\title{
More on Superconductors via Gauge/Gravity Duality with Nonlinear Maxwell Field
}

\author{
Davood Momeni, ${ }^{1}$ Muhammad Raza, ${ }^{2}$ and Ratbay Myrzakulov ${ }^{1}$ \\ ${ }^{1}$ Eurasian International Center for Theoretical Physics, Eurasian National University, Astana 010008, Kazakhstan \\ ${ }^{2}$ Department of Mathematics, COMSATS Institute of Information Technology (CIIT), Sahiwal Campus, Pakistan
}

Correspondence should be addressed to Davood Momeni; d.momeni@yahoo.com

Received 22 February 2013; Revised 16 March 2013; Accepted 16 March 2013

Academic Editor: Sergei Odintsov

Copyright (c) 2013 Davood Momeni et al. This is an open access article distributed under the Creative Commons Attribution License, which permits unrestricted use, distribution, and reproduction in any medium, provided the original work is properly cited.

\begin{abstract}
We have developed the recent investigations on the second-order phase transition in the holographic superconductor using the probe limit for a nonlinear Maxwell field strength coupled to a massless scalar field. By analytical methods, based on the variational Sturm-Liouville minimization technique, we study the effects of the spacetime dimension and the nonlinearity parameter on the critical temperature and the scalar condensation of the dual operators on the boundary. Further, as a motivated result, we analytically deduce the DC conductivity in the low and zero temperatures regime. Especially in the zero temperature limit and in two dimensional toy model, we thoroughly compute the conductivity analytically. Our work clarifies more features of the holographic superconductors both in different space dimensions and on the effect of the nonlinearity in Maxwell's strength field.
\end{abstract}

\section{Introduction}

In the recent years, using the holographic picture of the world, the AdS/CFT (anti de Sitter/conformal field theory) correspondence [1-3] has been applied to study some strongly correlated systems in condensed matter physics, especially for strongly coupled systems with the scaleinvariance. Particularly, people studied the low temperature, quantum critical systems near critical point (see, e.g., [4, 5] and references therein). The critical phenomena, which happen here, is a second-order phase transition from normal phase to the superconducting phase, in which below a specific temperature $T_{c}$, the DC conductivity becomes infinite. Such second-order phase transitions happen in the high-temperature superconductors and can be described very well by the AdS/CFT dictionary [6,7]. From the classical and phenomenological point of view, superconductivity, in the high-temperature type II superconductors, modeled by a phenomenological Landau-Ginzburg Lagrangian. This Lagrangian contains a general complex value scalar field $\Psi$, plays the role of a condensate in a superconductive phase. Basically, to have a scalar condensation in the boundary quantum field theory using CFT on the boundary of the bulk,
Hartnoll et al. [8] introduced a $U(1)$ abelian gauge field $A_{\mu}$ and a typical conformal coupled charged complex scalar field in the bulk black hole background. The conformal mass is above the Breitenlohner-Freedman (BF) bound [9]. To solve the negative mass problem, finally Gubser [10] showed that the vector potential modifies the mass term of scalar field and we have a possibility to have hairy black holes in some parts of the parameter space.

The full description of the superconductivity in the probe limit or away this limit needs to provide the numerical solutions of a couple of nonlinear differential equations. By simplicity, they can be solved using the shooting approach by expanding in series the functions and matching these by varying the free parameters of the series in a typical point between the horizon and the spatial infinity. Parallel to the numerical studies, recently some analytical approaches have been proposed to find the universal properties of secondorder phase transitions in holographic superconductors [1118]. In particular, the authors in [18] used the variational functional method on the real valued functions for the Sturm-Liouville eigenvalue problem to compute analytically the basic properties of the holographic superconductors in a three-dimensional boundary using the solutions of the 
bulk gauge fields in the four dimensional bulk theory. In $[18,19]$ the authors have shown that also it is possible to calculate critical exponent and critical temperature by this approach. The eigenvalue of this variational problem is a function of the critical chemical potential $\mu_{c}$, and consequently, it is related to the $T_{c}$. Different modes of super criticality s-wave, p-wave and d-wave have been studied [20]. Also, one can apply this method to superconductors with external magnetic fields, which gives the analytical results in good agreement with numerical results produced previously $[21,22]$.

Furthermore, a number of aspects of external and bulk magnetic fields in holographic superconductors have been investigated [23-26]. The phase transition can be interpreted in terms of the string interactions [27-31]. The effects of the nonlinear electrodynamics in the holographic superconductors have been investigated recently [32-36].

There are many interests in the modified gravity theories. For example, on Gauss-Bonnet and Weyl corrected superconductors, on which, we are working with a higher derivative corrected bulk black hole, like Weyl corrections [37] numerically. Furthermore, we have studied the Weyl corrections to the superconductors analytically [38, 39]. Moreover, we showed that there exists a family of p-wave holographic with Weyl corrections [40].

In the present paper, we would like to study the $((d-$ $2)+1)$-dimensional holographic superconductor in the probe limit for a power law Maxwell field strength $\left(F^{\mu \nu} F_{\mu \nu}\right)^{\delta}$ coupled to a scalar field. We focus just on the s-wave cases. We must clarify the motivation of the s-wave approximation in holographic models of superconductors. In the relativistic models of the gravity, it is highly known that s-wave approximation is not a good approximation, for example, in the cosmological models and black holes [41]. The meaning of the s-wave here does not back to the reduction of the action from four to two dimensional like the dilatonic action from the four dimensional spinor (Majorana) action. We mean by s-wave, in the context of holographic superconductors, a scalar order parameter, whose expectation value breaks the $U(1)$ but not rotational symmetry. Moreover, we can have the Yang-Mills fields with $S U(2)$ symmetry which additionally they can generate another symmetry breaking of an axial vector type. The last case resembles the p-wave models. We mention here that the three dimensional non-linear model of the superconductors, which we used in this paper, is a realistic model and it will be more interesting that we can find a direct relation between this nonlinear model and the results of a higher dimensional model, by a principle like the detailed balance.

Another additional point is to restrict ourselves just to the case of a single horizon. The problem of the multihorizon cases needs more investigation, for example, the case of the Nariai black holes. The theory here will be so different. This later appeared in the lower dimensional models. For example, the case of the quantum corrected BTZ like black hole is a good example [42]. In the holographic set up for superconductors one must identify a temperature in his gravitational bulk model to the CFT temperature on the boundary. If the black hole has only one horizon, in this case, we can use the Hawking-Bekenstein (horizon) or Kodama- Hayward temperature [43] as a reasonable candidate. But if our asymptotically AdS bulk has more than one horizon, for example, in the case of the charged BTZ like black holes, then we take the temperature of the real physical horizon (the temperature which is obtained by calculation the surface gravity of the biggest null hypersurface orthogonal surface) as the candidate for temperature of the CFT. In fact the effects of the quantum corrections and charged Maxwell field on the background of the bulk are very interesting problems and can be investigated in more details. Also it is possible to relate the instability of such charged dilaton configurations in the AdS spacetime [44] to the symmetry breaking mechanism of the superconductors. The idea has motivation enough as a new work. In this paper we investigate analytically the effect of the spacetime dimension $d$ and the power $\delta$ on the critical temperature $T_{c}$. Although our problem is the especial massless case of the model which has been investigated recently [45], we study these corrections to the superconductors analytically. Additionally, we want to compute the DC conductivity for this kind of the superconductor using the perturbation method. In this approach, we apply an external linear electromagnetic field. This field is periodic in time. By calculating, the response in the first order linear approximation, we compute the conductivity for the low temperature case, especially for zero temperature configuration.

Our plan in this paper is as the following. In Section 2, we clarify our motivation for considering the nonlinear Maxwell action instead of the linear theory. In Section 3, we introduce our model for holographic superconductors. In Section 4, we apply the variational method to obtain the critical temperature of the system. In Section 5, we calculate the critical exponent for the condensation operator. In Sections 6 and 7 we compute the conductivity for low and zero temperature cases. We summarize and conclude in the final section.

\section{Motivation for Nonlinear Maxwell Effects in Holographic Superconductors}

The linear approximations in the mathematical physics, as we know, have limitations, both in predictions of the model and especially on matching with the full description of the model using the numerical results. The linear Maxwell theory fails in some domains, and it is needed to consider the general form of the Lagrangian instead of the linear one. The Lagrangian of the Maxwell model is

$$
\mathscr{L}_{M}=-\frac{1}{4} F^{2},
$$

where $F^{2}=F^{\mu \nu} F_{\mu \nu}$. A natural extension of (1) is obtained by replacing a general function of $F$ in the form $\Phi(F)$. Recently, it has been shown that such nonlinear general forms have a rich family of black holes in $f(R)$ gravity [46]. There are different reasons for investigating these forms. The oldest one may be the Born-Infeld (BI) alternative for linear Maxwell's theory. 
In string theory language, this BI action can be replaced by the tachyonic action. The BI Lagrangian reads [47]

$$
\mathscr{L}_{\mathrm{BI}}=\eta^{2}\left(1-\sqrt{1+\frac{F^{2}}{2 \eta^{2}}}\right) .
$$

Here $\eta$ is the string's tension parameter. This form reduces to (1) in the limit of $\eta \rightarrow \infty$. Indeed, we can expand in series (2) in the following form:

$$
\mathscr{L}_{\mathrm{BI}}=\sum_{n=1}^{\infty} c_{n} \eta^{2(1-n)} F^{2 n} .
$$

The leading order term $n=1$ is just in the form of (1). Even, if we do not work with BI theory, this nonlinearity meets us from a geometrical point of view. Suppose that we want to write a conformal invariance (CI) Lagrangian, constructed from the $U(1)$ gauge fields $A^{\mu}$. Such CI is the invariance of the whole theory under geometrical transformation $g_{\mu \nu} \rightarrow$ $e^{2 \sigma} g_{\mu \nu}$ in a $d \geq 4$ Riemannian manifold without torsion or nonmetricity fields. However, previously, a version of such Lagrangian has been found in Weitznbock spacetime with torsion and nonmetricity fields [48]. It is easy to show that the Lagrangian $\mathscr{L} \propto F^{d / 2}$ is invariant under CI transformations. When $d=4$, the proper Lagrangian is $\mathscr{L} \propto F^{2}$ but in $d=5$ the suitable form is $\mathscr{L} \propto F^{5 / 2}$. The last form belongs to the nonlinear, noninteger Maxwell family. Thus from geometrical view, the nonlinearity is welcome in our Lagrangian dynamical theory. Further, even if we do not know any on BI or CI, when we are working with vacuum effects, there is a simple generalization of Maxwell Lagrangian in a logarithmic form

$$
\mathscr{L}_{\log }=-\eta^{2} \log \left(1+\frac{F^{2}}{4 \eta^{2}}\right) .
$$

As BI case, in the limit of $|F| \ll \eta$, by expanding this equation the leading order term $n=1$, is the linear theory (1). In brief, according to the above discussion, it seems that consideration of the nonlinear effects of the Maxwell field as $F^{\delta}$ is important, and the significant differences between the usual linear theory $F^{2}$ and nonlinear theory $F^{\delta}$ can be shown. Holographic superconductors provide a rich background for testing such type of new physics. In this paper, we will describe a ( $d-$ 1) dimensional holographic superconductor (HSC) via $d$ dimensional gravity dual, described by a $d$-dimensional AdS black hole on the static patch. We set the Stuckelberg field to zero and work with massless scalar fields.

\section{Field Equations}

We write the following action [32-36] for a power Maxwell field $F_{\mu \nu}$, which it is coupled minimally to a massless scaler field $\psi$ in a $d$-dimensional asymptotic $\mathrm{AdS}_{d}$ spacetime [45]

$$
S=\int d^{d} x \sqrt{-g}\left[R+(d-1)(d-2)-\xi\left(F^{\mu \nu} F_{\mu \nu}\right)^{\delta}-\left|D_{\mu} \psi\right|^{2}\right) .
$$

Here, we set the radius of the AdS, $L=1, D_{\mu}=\partial_{\mu}-i e A_{\mu}$, $F_{\mu \nu} \equiv 2 \partial_{[\mu} A_{\nu]}=\partial_{\mu} A_{\nu}-\partial_{\nu} A_{\mu}, A_{\mu}$ is $U(1)$ vector potential. The exponent (power) $\delta$ can be noninteger as we explained it in the previous section. Further, when we work with Maxwell linear electrodynamics $\delta=1, d=4$, then $\xi=1 / 4$. However, in our case with $\delta \neq 1$, in general, $\xi \in \mathscr{R}$ remains as a free parameter in our model. We set the electric charge $e=1$. In normal phase and in the absence of the scalar field we put $\psi=0$. This action has been used before in literature for full description of the nonlinear Maxwell field in the gravitational action. In the probe limit when the matter action and the gravitational part decouple, the system of the field equations has an exact solution which will be discussed here. The solution of the generalized Maxwell-Einstein equations is the simple $d$-dimensional static Reissner-Nordstrom-Antide Sitter (RNAdS) black hole with the following metric form:

$$
g_{\mu \nu}=\operatorname{diag}\left(-f(r), \frac{1}{f(r)}, r^{2} \Sigma_{d-2}\right),
$$

where $\Sigma_{d-2}$ is the metric on a $(d-2)$ dimensional sphere and the metric function is

$$
f(r)=r^{2}\left(1-\left(\frac{r_{+}}{r}\right)^{d-1}\right)
$$

Here $r_{+}$is the black hole horizon which in general is the largest root of the algebraic equation $f\left(r_{+}\right)=0$. If we set $\delta=1$ in (5), we recover the usual holographic superconductors. In the probe limit by ignoring the backreaction effects of the matter fields in the background metric $g_{\mu \nu}$, and with spherically symmetric static metric and by choosing a suitable gauge fixing for the gauge field $A_{\mu}$ we can take the functions $(\psi, \phi) \in \mathscr{R}$ and one variable functions. We assume that the functions $\psi$ and $\phi$ have finite numbers of poles on the real axis. It means the analytical solutions can be written in the forms of the algebraic series expressions. To obtain the field equations, we assume that $A_{\mu}=\phi(r) \delta_{\mu t}$, so the nonzero components of the $F_{\mu \nu}$ read as

$$
F_{r t}=-F_{t r}=\phi^{\prime}
$$

Hence, we have

$$
F^{\mu \nu} F_{\mu \nu}=-2 \phi^{\prime 2}
$$

Also, the Ricci $R$ of a $d$-dimensional spacetime with spherical symmetry reads

$$
R=-\left(f^{\prime \prime}+\frac{2(d-2) f^{\prime}}{r}-\frac{(d-2)(d-3) f}{r^{2}}\right) .
$$

Further, we compute

$$
\left|D_{\mu} \psi\right|^{2}=f \psi^{\prime 2}-\frac{\phi^{\prime 2}}{f}
$$


Finally, by plugging the above expressions into the action (5), making a partial integration, we get the following effective Lagrangian:

$$
\begin{aligned}
\mathscr{L}=r^{d-2}[ & -\left(f^{\prime \prime}+\frac{2(d-2) f^{\prime}}{r}-\frac{(d-2)(d-3) f}{r^{2}}\right) \\
& \left.+(d-1)(d-2)-\xi\left(-2 \phi^{\prime 2}\right)^{\delta}-\left(f \psi^{\prime 2}-\frac{\phi^{\prime 2}}{f}\right)\right] .
\end{aligned}
$$

As a first step, it is necessary to eliminate the $f^{\prime \prime}$ term, by integration part by part. After it, to write the field equation, we use the Euler-Lagrange equation as the following:

$$
\frac{d}{d r}\left(\frac{\partial \mathscr{L}}{\partial q_{, r}}\right)=\frac{\partial \mathscr{L}}{\partial q}, \quad q=\{\phi, \psi\}
$$

The field equations, derived from (12), read

$$
\begin{gathered}
\psi^{\prime \prime}+\left(\frac{f^{\prime}}{f}+\frac{d-2}{r}\right) \psi^{\prime}+\frac{\phi^{2}}{f^{2}} \psi=0, \\
\phi^{\prime \prime}+\frac{d-2}{2 \delta-1} \frac{\phi^{\prime}}{r}-C_{\delta} \frac{\psi^{2}}{\xi f} \phi\left(\phi^{\prime}\right)^{2(1-\delta)}=0,
\end{gathered}
$$

here $C_{\delta}=\left((-2)^{2-\delta} \delta(2 \delta-1)\right)^{-1}$. To avoid the pure complex numbers in our field equations, we assume that $\delta \neq 2-(1 / 2 N)$. The case with $\delta=1, D=4$ is the usual four-dimensional HSC which describes the three dimensional superconductors.

These field equations are the special massless case of the model which has been investigated recently $[45,49]$. To avoid the divergence near the singularity $f\left(r_{+}\right)=0$ we write the boundary conditions for (14), (15) by

$$
\phi\left(r_{+}\right)=\psi^{\prime}\left(r_{+}\right)=0 .
$$

The asymptotic solutions for system (14), (15) on the AdS boundary $r \rightarrow \infty$, are

$$
\begin{gathered}
\psi=D_{-}+\frac{D_{+}}{r^{d-1}}, \\
\phi=A-\frac{B}{r^{\eta}},
\end{gathered}
$$

in (18), $D_{ \pm}=\left\langle\mathcal{O}_{ \pm}\right\rangle, \eta=(d-2 \delta-1) /(2 \delta-1)$, where $\left\langle\mathcal{O}_{ \pm}\right\rangle$ denotes the vacuum expectation value of the dual operator $\mathcal{O}_{ \pm}$on the boundary, and the chemical potential and charge density of the dual theory are $A=\mu, B=\rho^{(2 \delta-1)^{-1}}$, respectively.

We must clarify the reason for the modification of the form of the electric potential $\phi$ modified. In the asymptotic regime, we know that the metric function $f$ behaves like $f \sim r^{2}$. Also, the scalar field has the following asymptotic form $\psi \sim 0$, so (15) gives us

$$
\phi_{\infty}^{\prime \prime} \sim-\frac{d-2}{2 \delta-1} \frac{\phi_{\infty}^{\prime}}{r} .
$$

The solution (19) reads

$$
\phi_{\infty}(r)=c_{1}+\frac{c_{0}(2 \delta-1)}{2 \delta-d+1} \frac{1}{r^{(d-2 \delta-1) /(2 \delta-1)}} .
$$

This solution coincides completely with the solution presented in (18). We mention here that the above function $\phi_{\infty}(r)$ in the limit of the linear electrodynamic theory $\delta=1$ has the true asymptotic form of $\phi_{\infty}(r) \sim r^{3-d}, d \neq 2$. For $d=2$ the expression of $\phi_{\infty}(r)$ is in the form of a diverging log term $\phi_{\infty}(r) \sim \log (r)$ and the application of the AdS/CFT fails. At least, we do not know the unique and true dictionary of the AdS/CFT in this lower dimensional bulk theory.

The only thing left is to identify the parameters with the physical quantities in the dual theory, that is, the chemical potential $\mu$ and the charge density $\rho$. We did it before, so the asymptotic behaviors have the same forms.

The asymptotic solutions for $\phi, \psi$ are the same as the previous expressions which have been presented in [45]. For normalization purposes, we set $D_{-}=0$.

\section{Variational Method}

To solve the solutions of the field equations given by (14) and (15), the well-known technique is numerical algorithms. However, from these numerical solutions, it is not so easy and straightforward to read $\langle\mathcal{O}\rangle$. Another method is using the matching method. It is a potentially powerful method. Even so, the results must be interpreted very carefully near the boundaries. The first step for solving system (14), (15) using variational approach [18], is rewriting the equations in a new dimensionless coordinate $z=r_{+} / r$ in the following forms:

$$
\begin{aligned}
& \psi^{\prime \prime}+\left(\frac{f^{\prime}}{f}-\frac{d-4}{z}\right) \psi^{\prime}+\left(\frac{r_{+}}{z^{2}}\right)^{2} \frac{\phi^{2}}{f^{2}} \psi=0, \\
& \phi^{\prime \prime}-\frac{\eta-1}{z} \phi^{\prime}-C_{\delta} \frac{r_{+}^{2 \delta}}{\xi z^{4 \delta}} \frac{\psi^{2}}{f} \phi\left(\phi^{\prime}\right)^{2(1-\delta)}=0 .
\end{aligned}
$$

Now, the prime is for differentiation with respect to the dimensionless radial coordinate $z$. Near the critical point $T=$ $T_{c}$, the following solution is valid:

$$
\phi \approx \phi_{B}\left(1-z^{\eta}\right) .
$$

Here $\phi_{B}=\mu_{c}=\rho^{(2 \delta-1)^{-1}} /\left(r_{+}\right)^{\eta+1}$ is the value of the $\phi$ at the horizon $r=r_{+}, \delta \neq(d-1) / 2$ and the critical point corresponds by the critical chemical potential $\mu=\mu_{c}$. Further, we can write

$$
\psi \approx\left\langle O_{+}\right\rangle\left(\frac{z}{r_{+}}\right)^{d-1} F(z)
$$

near the AdS boundary $z \rightarrow 0$ with $F(0)=1, F^{\prime}(0)=0$. The trial variational function satisfies the following second-order Sturm-Liouville self sdjoint differential equation:

$$
\left[\mu(z) F^{\prime}(z)\right]^{\prime}-Q(z) F(z)+\left(\frac{\phi_{B}}{r_{+}}\right)^{2} P(z) F(z)=0,
$$


where

$$
\begin{gathered}
\mu(z)=z^{2 d-2}\left(z^{d-1}-1\right), \\
P(z)=\mu(z)\left(\frac{1-z^{\eta}}{1-z^{d-1}}\right)^{2}, \\
Q(z)=-\mu(z)\left[\frac{(d-1)(d-2)}{z^{2}}-\frac{d-1}{z}\right. \\
\left.\times\left(\frac{2+(d-3) z^{d-1}}{z\left(1-z^{d-1}\right)}+\frac{d-4}{z}\right)\right],
\end{gathered}
$$

our strategy is to obtain the minimum value of $\phi_{B} / r_{+}$from the minimization of the following functional:

$$
\Theta(\delta, d) \equiv\left(\frac{\phi_{B}}{r_{+}}\right)_{\text {Min }}^{2}=\frac{\int_{0}^{1}\left(\mu(z) F^{\prime}(z)^{2}+Q(z) F^{2}(z)\right) d z}{\int_{0}^{1} P(z) F^{2}(z) d z} .
$$

The minimum of the critical temperature $T_{c}$ is obtained from the $T=(d-1) r_{+} / 4 \pi$. It reads

$$
T_{c}=\gamma \rho^{\eta+1},
$$

and $\gamma=((d-1) / 4 \pi)\left(\left(\phi_{B} / r_{+}\right)_{\text {Min }}\right)^{-(\eta+1)}$. Here we take trial function $F(z)=1-\alpha z^{2}$ in (27). It is useful to set $\rho=1$. The values of the critical temperature $T_{c}$ for $d=4,5$, by minimizing the functional (27) with trial function $F(z)$ and using (28) (in case $\rho=1$ ) are given by the following.

For $d=4, \delta=1: T_{c}=0.0844$, for $\delta=3 / 4, T_{c}=$ 0.1692 .

For $d=5, \delta=1: T_{c}=0.01676$, for $\delta=3 / 4, T_{c}=$ 0.2503 , for $\delta=5 / 4, T_{c}=0.0954$.

These values are in good agreements with the numerical values [50] and also coincide to the analytical values given in [45].

\section{Calculating the Critical Exponent}

We begin from (22) by writing it near the critical point (CP) and in limit $T \rightarrow T_{c}$. The first step is rewriting the solution in a perturbative scheme with respect to the perturbation parameter $\epsilon=\left\langle O_{+}\right\rangle^{2}$, as it was described by Kanno [51]. Near the $\mathrm{CP}$, the solution of the field $\phi$ is written as

$$
\phi(z)=\phi_{0}+\epsilon \chi(z),
$$

where $\phi_{0}=\kappa T_{c}\left(1-z^{\eta}\right)$. Using (24) and (29) in first order with respect to the $O(\epsilon)$ we obtain the following ordinary differential equation:

$$
\chi^{\prime \prime}(z)-\frac{\eta-1}{z} \chi^{\prime}(z)=E(z),
$$

where

$$
E(z)=\frac{C_{\delta} r_{+}^{2} z^{-\delta-2} F^{2}(z)}{\xi f(z)} \phi_{0}^{2}\left(\phi_{0}\right)^{\prime 2(1-\delta)},\left.\quad F(z)\right|_{z \rightarrow 0} \approx 1 .
$$

Since $\phi(z)=\left(A\left(T_{c}\right)^{\eta+1} / T^{\eta}\right)\left(1-z^{\eta}\right), A=(d-1) / 4 \pi$ writing the solution for $\phi$ in $z=0$, we have

$$
\phi(0)=\phi_{0}(0)+\epsilon \chi(0) .
$$

The general solution for (30) is given by

$$
\begin{aligned}
\chi(z)= & \sqrt{z}\left(c_{1} J_{1}(x)+c_{2} Y_{1}(x)\right) \\
& +\sqrt{z} \pi \eta^{2-2 \delta} T_{c}{ }^{4-2 \delta} \kappa^{4-2 \delta} f(x),
\end{aligned}
$$

with

$$
\begin{aligned}
f(x)= & \alpha J_{1}(x) \int \frac{Y_{1}(x)\left(z^{\eta}-1\right)^{2} z^{2 \eta-1 / 2+\delta-2 \delta \eta}}{z^{d}-z} d z \\
& +\beta Y_{1}(x) \int \frac{J_{1}(x)\left(z^{\eta}-1\right)^{2} z^{2 \eta-1 / 2+\delta-2 \delta \eta}}{z^{d}-z} d z .
\end{aligned}
$$

Here, $x=2 \sqrt{(1-\eta) z},\left\{J_{n}(x), Y_{n}(x)\right\}$ are Bessel functions of first and second kinds.

Finding the value of $\chi(0)$ from (33), and solving it for $\left\langle O_{+}\right\rangle=\sqrt{\epsilon}$, we obtain (we take $T_{c}=1$ )

$$
\left\langle O_{+}\right\rangle \propto T^{d-\delta-\eta / 2}\left[1-T^{\eta}\right]^{1 / 2} .
$$

When the power $\delta$ decreases, the value of the $\left\langle\mathrm{O}_{+}\right\rangle$ increases. Thus we conclude that the effect of the power $\delta$ in $d=4$ model is in the direction of the increase of $\left\langle O_{+}\right\rangle$.

However, in the five dimensions, the analysis is a little bit different. As we observe, in $d=5$, when the power $\delta$ increases, the value of the $\left\langle O_{+}\right\rangle$increases. Thus, we can say that the effect of the power $\delta$ in $d=5$ model is in the direction of the increase of $\left\langle\mathrm{O}_{+}\right\rangle$.

\section{Calculating the Low Temperature DC Conductivity}

In this section, we compute the low-temperature DC conductivity. We concentrate on the general space time dimension $d$, and we will try to calculate the conductivity $\sigma$ as a function of the rescaled frequency as follows:

$$
\widehat{\omega}=\frac{\omega}{\left\langle O_{+}\right\rangle^{1 / \Delta}}, \quad \Delta=d-1 .
$$

In this limit, the asymptotic form of the scalar field is

$$
\psi(z)=\frac{b^{d-1}}{\sqrt{2}} z^{d-1} F(z), \quad b \equiv\left\langle O_{+}\right\rangle .
$$

We follow the method in [18]. Assuming that there exists an external magnetic field $A(r, t)=A(r) e^{-i \omega t}$. Note that here, the applied Maxwell field is linear. It is not related to the nonlinear Maxwell's field in the bulk action. In fact, the nonlinearity of the Maxwell field now is stored in the background metric. As we know, the field equations have some terms which involve the exponent $\delta$. This parameter denotes the nonlinearity, which is hidden in the structure of the background metric and through it. 
Moreover, it diffuses to the dynamics of the scalar field and the Abelian gauge field $U(1)$, so to compute the conductivity the applied external magnetic field is linear and satisfies the usual linear Maxwell field $F_{\mu \nu}^{; \mu}=0$, which is nothing but the linear wave equation.

The linear wave equation for this field reduces to

$$
-\frac{d^{2} A}{d \bar{r}^{2}}+V(r) A=\omega^{2} A .
$$

Equation (38) is written in the Schrodinger's form and in terms of the new tortoise coordinate $\bar{r}=$ $-\left(1 / r_{+}\right) \sum_{n=0}^{\infty}\left(z^{n(d-1)+1} /(n(d-1)+1)\right)$. The horizon $r=r_{+}$ is located at $z=1$ or $\bar{r} \rightarrow-\infty$. Here the potential is $V=2 f(r) \psi^{2}(r)$. The ingoing waves in horizon behave as a boundary condition (BC) for solving this wave equation (38) read as

$$
A \sim e^{-i \omega \bar{r}} \sim(1-z)^{-i \omega /(d-1) r_{+}} .
$$

The electromagnetic wave equation (38), in the coordinate $z$, reads

$$
A_{z z}+\left(\frac{2}{z}+\frac{f^{\prime}}{2 f}\right) A_{z}+\left(-\frac{2 r_{+}^{2} \psi(z)^{2}}{z^{4}}+\frac{\omega^{2} r_{+}^{2}}{z^{4} f}\right) A=0
$$

By replacing (37), we obtain

$$
\begin{gathered}
A_{z z}+p_{1}(z) A_{z}+Q_{1}(z) A=0, \\
p_{1}(z)=\left(\frac{2}{z}+\frac{f^{\prime}}{2 f}\right), \\
Q_{1}(z)=-\frac{r_{+}^{2} b^{2(d-1)} z^{2(d-1)} F^{2}(z)}{z^{4}}+\frac{\omega^{2} r_{+}^{2}}{z^{4} f} .
\end{gathered}
$$

To keep the boundary conditions, we put

$$
A=(1-z)^{-i \omega /(d-1) r_{+}} e^{-i \omega z /(d-1) r_{+}} \Theta(z)
$$

Substituting this ansatz in (41), we obtain

$$
\begin{gathered}
\Theta^{\prime \prime}+P(z) \Theta^{\prime}+Q(z) \Theta=0 \\
P(z)=\frac{r_{+}(1-z)(d-1) p_{1}(z)+2 i \omega z}{r_{+}(1-z)(d-1)} \\
Q(z)=\left(i z(1-z) \omega r_{+}(d-1) p_{1}(z)\right. \\
+r_{+}^{2}(d-1)^{2}(1-z)^{2} Q_{1}(z) \\
\left.+\omega\left(i(d-1) r_{+}-\omega z^{2}\right)\right) \\
\times\left(r_{+}^{2}(d-1)^{2}(1-z)^{2}\right)^{-1}
\end{gathered}
$$

By imposing the regularity condition on wave function $\Theta$ at the black hole horizon $z=1$, we obtain the following auxiliary boundary condition:

$$
\begin{aligned}
0=\Theta^{\prime}(1) \lim _{z \rightarrow 1}\left(\frac{1}{2} r_{+}(1-z)(d-1) p_{1}(z)+i \omega z\right) \\
+\Theta(1) \lim _{z \rightarrow 1}\left(i z(1-z) \omega r_{+}(d-1) p_{1}(z)\right. \\
+r_{+}^{2}(d-1)^{2}(1-z)^{2} Q_{1}(z) \\
\left.+\omega\left(i(d-1) r_{+}-\omega z^{2}\right)\right) .
\end{aligned}
$$

Explicitly, by computing the limits, we have

$$
i\left(-r_{+}+i \omega+r_{+} d\right) \omega \Theta(1)=0 \text {. }
$$

One possibility is $\Theta(1)=0$. Another $\omega=i r_{+}(d-1)$, but the last case from (39) leads to the

$$
A \sim e^{-i \omega \bar{r}} \sim,(1-z)
$$

which has no meaning as the ingoing wave toward the horizon, so we impose $\Theta(1)=0$. Since we are working in the low temperature limit, we take the limit $b \rightarrow \infty$, so we rescale the coordinate $z$ by $z \rightarrow z / b$. Also we must put one suitable trial form for $F(z)$, for the case of $\Delta=d-1>3 / 2, d>$ 2 we put $F(z / b) \rightarrow F(0)=1$. We rescale (43), so we obtain

$$
\Theta^{\prime \prime}+b P\left(\frac{z}{b}\right) \Theta^{\prime}+b^{2} Q\left(\frac{z}{b}\right) \Theta=0, \quad{ }^{\prime}=d / d(z / b) .
$$

Finally, we obtain

$$
\Theta^{\prime \prime}+\frac{z}{r_{+}^{2}}\left(1+\frac{2 i r_{+} \widehat{\omega}}{d-1}\right) \Theta^{\prime}+\frac{3 i \widehat{\omega}}{r_{+}(d-1)} \Theta=0 .
$$

The general solution for (50) reads

$$
\Theta(z)=z \mathrm{e}^{-z^{2}\left(1 / 2+i \widehat{\omega} r_{+}\right) / r_{+}^{2}}
$$

$$
\begin{array}{r}
\times\left(c_{+} M\left(\mu, v, \frac{1}{2} \frac{\left(1+2 i \widehat{\omega} r_{+}\right) z^{2}}{r_{+}^{2}}\right)\right. \\
\left.+c_{-} U\left(\mu, \nu, \frac{\left(1+2 i \widehat{\omega} r_{+}\right) z^{2}}{r_{+}^{2}}\right)\right)
\end{array}
$$

$$
\mu=\frac{1}{2} \frac{2 d-2+4 i \widehat{\omega} r_{+} d-7 i \widehat{\omega} r_{+}}{\left(1+2 i \widehat{\omega} r_{+}\right)(d-1)}, \quad v=\frac{3}{2} \text {. }
$$

The DC conductivity in the low temperature limit is defined by

$$
\sigma(\widehat{\omega})=\frac{i}{\widehat{\omega}} \frac{\Theta^{\prime}\left(-\widehat{\omega}^{2}\right)}{\Theta\left(-\widehat{\omega}^{2}\right)} .
$$

We need the asymptotic limit of (51). Indeed, we guess that $\lim _{z \rightarrow \infty} \Theta(z) \approx \Theta\left(-\widehat{\omega}^{2}\right)$ where here the $\widehat{\omega}$ is the quasinormal modes and locates on the real axis, so we guess $\mathfrak{R e}[\sigma(\widehat{\omega})]=0$ 
except at the poles of $\mathfrak{I} \mathfrak{m}[\sigma(\widehat{\omega})]$ where as $\mathfrak{R e}[\sigma(\widehat{\omega})] \approx \delta(\widehat{\omega})$. First, by imposing $\Theta(1)=0$ we have

$$
\begin{aligned}
& \Theta(z)=c_{-} \frac{z \mathrm{e}^{-z^{2}\left(1 / 2+i \widehat{\omega} r_{+}\right) / r_{+}^{2}}}{U\left(\mu, \nu,\left(1+2 i \widehat{\omega} r_{+}\right) / r_{+}^{2}\right)} \\
& \times\left[M\left(\mu, \nu, \frac{\left(1+2 i \widehat{\omega} r_{+}\right) z^{2}}{r_{+}^{2}}\right) U\left(\mu, \nu, \frac{1+2 i \widehat{\omega} r_{+}}{r_{+}^{2}}\right)\right. \\
&-U\left(\mu, \nu, \frac{\left(1+2 i \widehat{\omega} r_{+}\right) z^{2}}{r_{+}^{2}}\right) \\
&\left.\quad \times M\left(\mu, \nu, \frac{1+2 i \widehat{\omega} r_{+}}{r_{+}^{2}}\right)\right]
\end{aligned}
$$

Here $M(\mu, v, x), U(\mu, v, x)$ denote the Kummer functions [52].

The quasinormal modes are the solutions of the following equation:

$$
U\left(\mu, \nu, \frac{1+2 i \widehat{\omega} r_{+}}{r_{+}^{2}}\right)=M\left(\mu, \nu, \frac{1+2 i \widehat{\omega} r_{+}}{r_{+}^{2}}\right)
$$

which has no closed, analytical solution and can be solved just numerically.

\section{Calculating the Zero Temperature Conductivity}

In this section, we compute analytically the conductivity $\sigma(\widehat{\omega})$ in the zero temperature $T=0$ limit. This case corresponds to the limiting case $b \rightarrow \infty$. We begin by writing (38) in the coordinate $z^{\prime}=z / b$

$$
\begin{aligned}
A_{z^{\prime} z^{\prime}}+\frac{1}{2 z^{\prime}}\left[\frac{2-(d+1)\left(b z^{\prime}\right)^{d-1}}{1-\left(b z^{\prime}\right)^{d-1}}\right] \\
\times A_{z^{\prime}}+\frac{r_{+}^{2}}{\left(b z^{\prime}\right)^{4}}\left[\frac{b^{2} z^{\prime 2} \widehat{\omega}^{2}}{1-\left(b z^{\prime}\right)^{d-1}}\right. \\
\left.\quad-\left(b^{2} z^{\prime}\right)^{2(d-1)} F\left(b z^{\prime}\right)^{2}\right] A=0 .
\end{aligned}
$$

By taking the limit $b \rightarrow \infty$ we have

$$
A_{z^{\prime} z^{\prime}}+\frac{d+1}{2 z^{\prime}} A_{z^{\prime}}+r_{+}^{2}\left[-\frac{\widehat{\omega}^{2}}{\left(b z^{\prime}\right)^{d+1}}-b^{4(d-2)} z^{\prime 2(d-3)}\right] A=0 .
$$

In (57) we set $\lim _{b \rightarrow \infty} F\left(b z^{\prime}\right)=F(0)=1$. The closed form of the exact solution for (57) depends on the value of the $d$.
Below we list the solutions for special dimensions $d=2, d=3$ as follows:

$$
\begin{aligned}
A\left(z^{\prime}\right) & =C z^{1-1 / 4} N_{-\sqrt{1+16 r_{+}^{2} / 2}}\left(\frac{2 i \widehat{\omega} r_{+}}{b^{3 / 2} \sqrt{z^{\prime}}}\right), \quad d=2 \\
A\left(z^{\prime}\right)= & \frac{H\left(0, \beta, \delta, \gamma,\left(z^{\prime 2}+1\right) /\left(z^{\prime 2}-1\right)\right)}{\sqrt{z^{\prime}}} \\
& \times\left(c_{1}+c_{2} \int \frac{d z^{\prime}}{z H\left(0, \beta, \delta, \gamma,\left(z^{\prime 2}+1\right) /\left(z^{\prime 2}-1\right)\right)}\right), \\
d=3 . & =
\end{aligned}
$$

Here

$$
\begin{gathered}
\beta=-\frac{1}{4} \frac{4 b^{8} r_{+}^{2}+b^{4}+4 r_{+}^{2} \widehat{\omega}^{2}}{b^{4}}, \\
\delta=-2 \frac{r_{+}^{2}\left(b^{8}-\widehat{\omega}^{2}\right)}{b^{4}}, \\
\gamma=-\frac{1}{4} \frac{4 r_{+}^{2} \widehat{\omega}^{2}-b^{4}+4 b^{8} r_{+}^{2}}{b^{4}} .
\end{gathered}
$$

Here $N_{v}(x)$ is the second kind of the Bessel function and $H(0, \beta, \delta, \gamma, x)$ denotes the Heun double confluent function [53], by expending (58), (59) in series in the form

$$
A\left(z^{\prime}\right)=A_{0}+A_{2}\left(b z^{\prime}\right)^{2}-\cdots
$$

The conductivity can be computed via the following simple formula:

$$
\sigma(\widehat{\omega})=\frac{2}{i \widehat{\omega}} \frac{A_{2}}{A_{0}}+\frac{i \widehat{\omega}}{2} .
$$

So by computing the asymptotic series, the conductivity will be determined by the analytical expression.

It is appropriate here to present the explicit form of the conductivity for one case. We choose the case given by (58). In the zero temperature limit, we treat the horizon size very tiny, so in application we take $\sqrt{1+16 r_{+}^{2}} \approx 1$. Also, it is better we define $x=2 \widehat{\omega} r_{+} / b^{3 / 2} \sqrt{z^{\prime}}$, which by the definition of the $z^{\prime}$ reads as $x=2 \omega r_{+} / b \sqrt{z}$. When $b \rightarrow \infty$, then $x \ll 1$, so, we can expand the Bessel function in terms of the infinitesimal argument $x$, and hence rewrite (58) as the following form:

$$
A(z)=C \sqrt[4]{\frac{b}{z}} N_{-v}(i x), \quad v=\frac{1}{2}, x=\frac{2 \omega r_{+}}{b \sqrt{z}}
$$

Remembering the following identities

$$
N_{-\nu}(i x)=\frac{i^{-\nu-1} I_{-\nu}(x) \cosh (\nu x)-i^{\nu-1} I_{\nu}}{\sinh (\nu x)}
$$




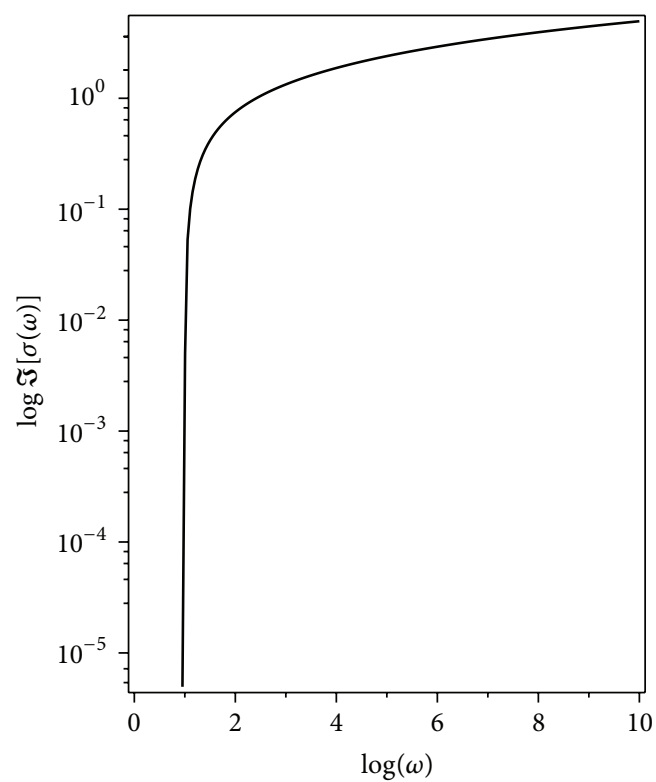

FIGURE 1: Variation of the $\log (\mathfrak{\Im m}[\sigma(\widehat{\omega})])$ for zero temperature limit in $d=2$.

Also, we know that

$$
I_{1 / 2}(x)=\sqrt{\frac{2}{\pi x}} \cosh (x), \quad I_{-(1 / 2)}(x)=\sqrt{\frac{2}{\pi x}} \sinh (x),
$$

so we have

$$
N_{-(1 / 2)}(i x)=\sqrt{\frac{2}{i \pi x}}\left[\frac{i \cosh (x) \cosh (x / 2)-\sinh (x)}{\sinh (x / 2)}\right] .
$$

Also, in limit $x \ll 1$,we have

$$
\begin{aligned}
& \frac{i \cosh (x) \cosh (x / 2)-\sinh (x)}{\sinh (x / 2)} \\
& =\frac{2 i}{x}-2+\frac{7}{6} i x-\frac{1}{4} x^{2}+\frac{59}{360} i x^{3}+O\left(x^{4}\right) .
\end{aligned}
$$

So for the electromagnetic field we have

$$
A(z) \approx C \frac{\sqrt[4]{b}}{\sqrt{i \pi r_{+} \widehat{\omega}}}\left[\frac{i \sqrt{z}}{\widehat{\omega} r_{+}}-2+\frac{7}{3} i \frac{\widehat{\omega} r_{+}}{\sqrt{z}}-\frac{\widehat{\omega}^{2} r_{+}^{2}}{z}\right] .
$$

Finally, we obtain the conductivity using (62) by the following simple formula:

$$
\sigma(\widehat{\omega})=\frac{1}{2 i \widehat{\omega}}+\frac{i \widehat{\omega}}{2}
$$

where as we guess $\mathfrak{R} \mathfrak{e}[\sigma(\widehat{\omega})]=0$. Figure 1 shows $\mathfrak{I} \mathfrak{m}[\sigma(\widehat{\omega})]$ as a function of the $\widehat{\omega}$ for zero temperature case and in $d=$ 2. Equation (69) gives the expression for DC conductivity in the zero temperature limit in $\mathrm{AdS}_{2} / \mathrm{CFT}_{1}$ model. Indeed, it is comparable with the numerical results of the previous papers about one dimensional holographic superconductors [54].

\section{Conclusion}

In this paper, we investigated the analytical properties of a holographic superconductor with power Maxwell's field. We studied the problem in the probe limit. We observed that it is possible to find the critical temperature $T_{c}$ and the condensation $\left\langle\mathrm{O}_{+}\right\rangle$and the conductivity $\sigma(\omega)$ via SturmLiouville variational approach. We concluded that in $d=4$, when the power $\delta$ decreases, the value of the $\left\langle\mathrm{O}_{+}\right\rangle$increases. Thus we can say that the effect of the power $\delta$ in $d=4$ model is in the direction of the increase of $\left\langle O_{+}\right\rangle$. In $d=5$ when the power $\delta$ increases, the value of the $\left\langle\mathrm{O}_{+}\right\rangle$increases. Thus, we can say that the effect of the power $\delta$ in $d=5$ model is in the direction of the increase of $\left\langle\mathrm{O}_{+}\right\rangle$. Further, we analytically deduced the low temperature and the zero temperature DC conductivity $\sigma$ as a function of the $\widehat{\omega}=\omega /\left\langle O_{+}\right\rangle^{1 / \Delta}$. Our work helps to give a better understanding of some unfamiliar effects of the holographic superconductors both in different space dimensions and nonlinearity in Maxwell's strength field.

\section{References}

[1] J. M. Maldacena, "The large $N$ limit of superconformal field theories and supergravity," Advances in Theoretical and Mathematical Physics, vol. 2, pp. 231-252, 1998.

[2] S. S. Gubser, I. R. Klebanov, and A. M. Polyakov, "Gauge theory correlators from non-critical string theory," Physics Letters, Section B, vol. 428, no. 1-2, pp. 105-114, 1998.

[3] E. Witten, "Anti-de sitter space and holography," Advances in Theoretical and Mathematical Physics, vol. 2, pp. 253-291, 1998.

[4] C. P. Herzog, "Lectures on holographic superfluidity and superconductivity," Journal of Physics A, vol. 42, no. 34, Article ID 343001, 2009.

[5] S. A. Hartnoll, "Lectures on holographic methods for condensed matter physics," Classical and Quantum Gravity, vol. 26, no. 22, Article ID 224002, 2009.

[6] G. Policastro, D. T. Son, and A. O. Starinets, "Shear viscosity of strongly coupled $N=4$ supersymmetric yang-mills plasma," Physical Review Letters, vol. 87, no. 8, Article ID 081601, 4 pages, 2001.

[7] P. Kovtun, D. T. Son, and A. O. Starinets, "Holography and hydrodynamics: diffusion on stretched horizons," Journal of High Energy Physics, vol. 7, no. 10, pp. 1675-1701, 2003.

[8] S. A. Hartnoll, C. P. Herzog, and G. T. Horowitz, "Building a holographic superconductor," Physical Review Letters, vol. 101, no. 3, Article ID 031601, 4 pages, 2008.

[9] P. Breitenlohner and D. Z. Freedman, "Stability in gauged extended supergravity," Annals of Physics, vol. 144, no. 2, pp. 249-281, 1982.

[10] S. S. Gubser, "Breaking an abelian gauge symmetry near a black hole horizon," Physical Review D, vol. 78, Article ID 065034, 15 pages, 2008.

[11] X. H. Ge, B. Wang, S. F. Wu, and G. H. Yang, "Analytical study on holographic superconductors in external magnetic field," Journal of High Energy Physics, vol. 1008, p. 108, 2010.

[12] H. F. Li, R. G. Cai, and H. Q. Zhang, "Analytical studies on holographic superconductors in Gauss-Bonnet gravity," Journal of High Energy Physics, vol. 1104, p. 028, 2011. 
[13] R. G. Cai, H. F. Li, and H. Q. Zhang, "Analytical studies on holographic insulator/superconductor phase transitions," Physical Review D, vol. 83, no. 12, Article ID 126007, 7 pages, 2011.

[14] C. M. Chen and M. F. Wu, "An analytic analysis of phase transitions in holographic superconductors," Progress of Theoretical Physics, vol. 126, pp. 387-395, 2011.

[15] X. H. Ge and H. Q. Leng, "Analytical calculation on critical magnetic field in holographic superconductors with backreaction," Progress of Theoretical Physics, vol. 128, pp. 1211-1228, 2012.

[16] M. R. Setare, D. Momeni, R. Myrzakulov, and M. Raza, "Holographic superconductors in the AdS black hole with a magnetic charge," Physica Scripta, vol. 86, Article ID 045005, 2012.

[17] D. Momeni, M. R. Setare, and N. Majd, "Holographic superconductors in a model of non-relativistic gravity," Journal of High Energy Physics, vol. 05, p. 118, 2011.

[18] G. Siopsis, J. Therrien, and S. Musiri, "Holographic superconductors near the Breitenlohner-Freedman bound," Classical and Quantum Gravity, vol. 29, no. 8, Article ID 085007.

[19] H. B. Zeng, X. Gao, Y. Jiang, and H. S. Zong, "Analytical computation of critical exponents in several holographic superconductors," Journal of High Energy Physics, 2011.

[20] S. Gangopadhyay and D. Roychowdhury, "Analytic study of properties of holographic p-wave superconductors," Journal of High Energy Physics, vol. 08, p. 104, 2012.

[21] E. Nakano and W. Y. Wen, "Critical magnetic field in a holographic superconductor," Physical Review D, vol. 78, no. 4, Article ID 046004, 3 pages, 2008.

[22] T. Albash and C. V. Johnson, "A holographic superconductor in an external magnetic field," Journal of High Energy Physics, vol. 2008, p. 121, 2008.

[23] O. Domenech, M. Montull, A. Pomarol, A. Salvio, and P. J. Silva, "Emergent gauge fields in holographic superconductors," Journal of High Energy Physics, vol. 2010, p. 033, 2010.

[24] M. Montull, O. Pujolas, A. Salvio, and P. J. Silva, "Flux periodicities and quantum hair on holographic superconductors," Physical Review Letters, vol. 107, no. 18, Article ID 181601, 2011.

[25] M. Montull, O. Pujolas, A. Salvio, and P. J. Silva, "Magnetic response in the holographic insulator/superconductor transition," Journal of High Energy Physics, vol. 1204, p. 135, 2012.

[26] Salvio A, "Holographic superfluids and superconductors in dilaton-gravity," Journal of High Energy Physics, vol. 1209, p. 134, 2012.

[27] N. Bobev, A. Kundu, K. Pilch, and N. P. Warner, "Minimal holographic superconductors from maximal supergravity," Journal of High Energy Physics, 25 pages, 2011.

[28] N. Bobev, N. Halmagyi, K. Pilch, and N. P. Warner, "Supergravity instabilities of non-supersymmetric quantum critical points," Journal of High Energy Physics, 46 pages, 2010.

[29] N. Bobev, A. Kundu, K. Pilch, and N. P. Warner, "Minimal holographic superconductors from maximal supergravity," Journal of High Energy Physics, 25 pages, 2012.

[30] T. Fischbacher, K. Pilch, and N. P. Warner, "New supersymmetric and stable, non-supersymmetric phases in supergravity and holographic field theory," http://arxiv.org/abs/1010.4910.

[31] N. Bobev, A. Kundu, K. Pilch, and N. P. Warner, "Minimal holographic superconductors from maximal supergravity," Journal of High Energy Physics, 2011.

[32] D. Roychowdhury, "AdS/CFT superconductors with power maxwell electrodynamics: reminiscent of the meissner effect," Physics Letters B, vol. 718, no. 3, pp. 1089-1094, 2013.
[33] D. Roychowdhury, "Effect of external magnetic field on holographic superconductors in presence of nonlinear corrections," Physical Review D, vol. 86, no. 10, Article ID 106009, 12 pages, 2012.

[34] R. Banerjee, S. Gangopadhyay, D. Roychowdhury, and A. Lala, "Holographic $s$-wave condensate with non-linear electrodynamics: a nontrivial boundary value problem," http://arxiv.org/abs/1208.5902.

[35] S. Gangopadhyay and D. Roychowdhury, "Analytic study of gauss-bonnet holographic superconductors in born-Infeld electrodynamics," Journal of High Energy Physics, vol. 05, p. 156, 2012.

[36] S. Gangopadhyay and D. Roychowdhury, "Analytic study of properties of holographic superconductors in born-infeld electrodynamics," Journal of High Energy Physics, vol. 05, p. 002, 2012.

[37] J. P. Wu, Y. Cao, X. M. Kuang, and W. J. Li, "The 3+1 holographic superconductor with Weyl corrections," Physics Letters B, vol. 697, no. 2, pp. 153-158, 2011.

[38] D. Momeni and M. R. Setare, "A note on holographic superconductors with Weyl corrections," Modern Physics Letters A, vol. 26, no. 38, p. 2889, 2011.

[39] D. Momeni, M. R. Setare, and R. Myrzakulov, "Condensation of the scalar field with stuckelberg and weyl corrections in the background of a planar AdS-schwarzschild black hole," International Journal of Modern Physics A, vol. 27, Article ID 1250128, 2012.

[40] D. Momeni, N. Majd, and R. Myrzakulov, " $p$-wave holographic superconductors with Weyl corrections," Europhysics Letters, vol. 97, no. 6, Article ID 61001, 2012.

[41] S. Nojiri and S. D. Odintsov, "Anomaly-induced effective actions in even dimensions and reliability of $s$-wave approximation," Physics Letters B, vol. 463, no. 1, Article ID 990414, pp. 57-62, 1999.

[42] S. Nojiri and S. D. Odintsov, "Can quantum-corrected btz black hole anti-evaporate?” Modern Physics Letters A, vol. 13, no. 33, Article ID 980603, p. 2695, 1998.

[43] S. A. Hayward, "Unified first law of black-hole dynamics and relativistic thermodynamics," Classical and Quantum Gravity, vol. 15, no. 10, p. 3147, 1998.

[44] S. Nojiri and S. D. Odintsov, "Quantum (in)stability of 2D charged dilaton black holes and 3D rotating black holes," Physical Review D, vol. 59, no. 4, Article ID 044003, 11 pages, 1999.

[45] J. Jing, Q. Pan, and S. Chen, "Holographic superconductors with Power-Maxwell field," Journal of High Energy Physics, vol. 2011, p. 045, 2011.

[46] S. H. Mazharimousavi and M. Halilsoy, "Black hole solutions in $\mathrm{f}(\mathrm{R})$ gravity coupled with non-linear Yang-Mills field," Physical Review D, vol. 84, Article ID 064032, 2011.

[47] M. Born and L. Infeld, "Foundations of the new field theory," Proceedings of the Royal Society A, vol. 144, no. 852, pp. 425-451, 1934.

[48] Y. N. Obukhov, "Conformal invariance and space-time torsion," Physics Letters A, vol. 90, no. 1-2, pp. 13-16, 1982.

[49] Q. Pan, J. Jing, and B. Wang, "Holographic superconductor models with the Maxwell field strength corrections," Physical Review D, vol. 84, 2011.

[50] G. T. Horowitz and M. M. Roberts, "Holographic superconductors with various condensates," Physical Review D, vol. 78, no. 12, Article ID 126008, 8 pages, 2008. 
[51] S. Kanno, "A note on gauss-bonnet holographic superconductors," Classical and Quantum Gravity, vol. 28, no. 12, Article ID 127001, 2011.

[52] M. Abramowitz and I. Stegun, Handbook of Mathematical Functions, Dover, New York, NY, USA, 1972.

[53] A. Ronveaux, Heun's Differential Equations, Oxford University Press, 1995.

[54] J. Ren, "One-dimensional holographic superconductor from $\mathrm{AdS}_{3} / \mathrm{CFT}_{2}$ correspondence," Journal of High Energy Physics, vol. 1011, p. 055, 2010. 

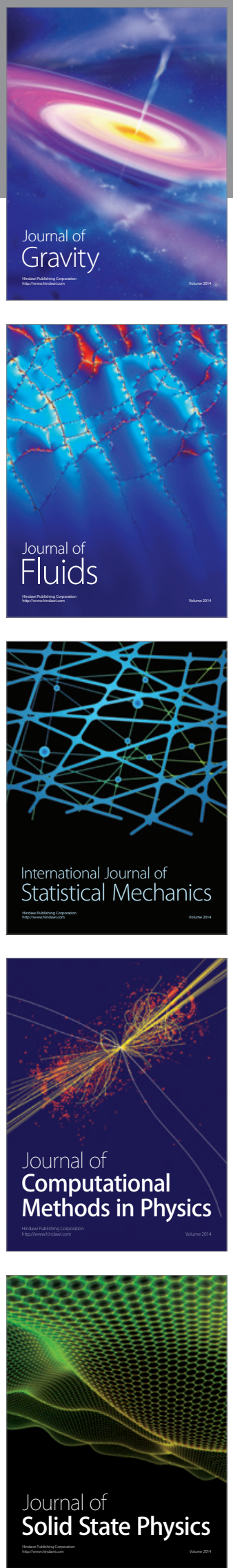

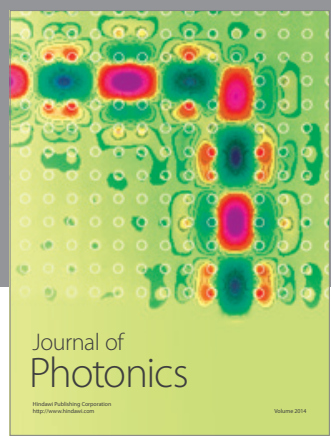

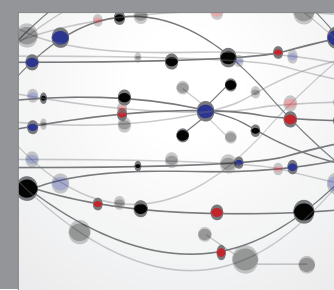

The Scientific World Journal

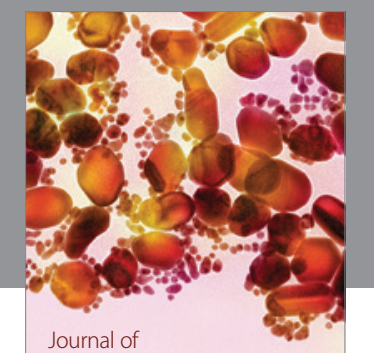

Soft Matter
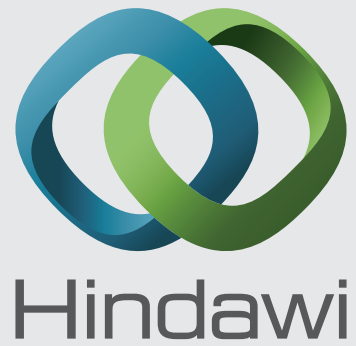

Submit your manuscripts at

http://www.hindawi.com
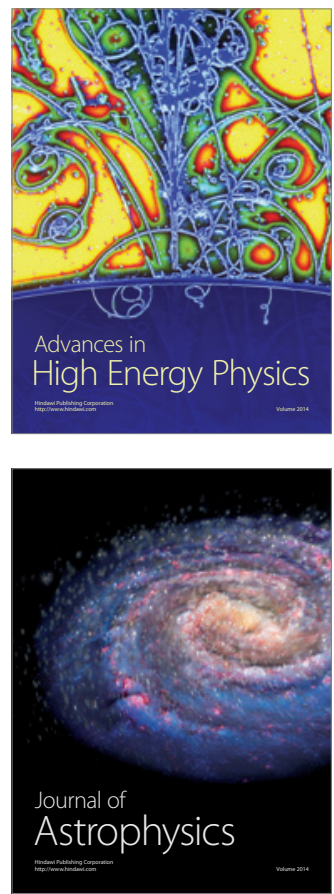
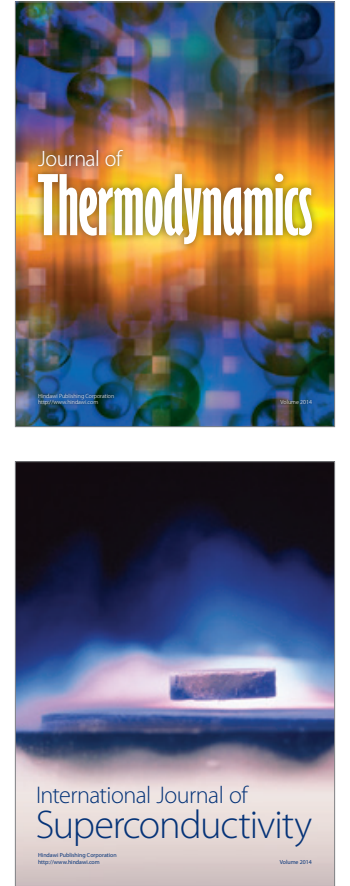
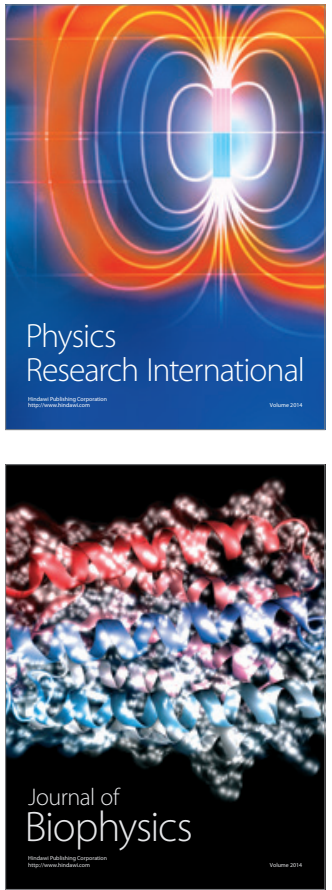
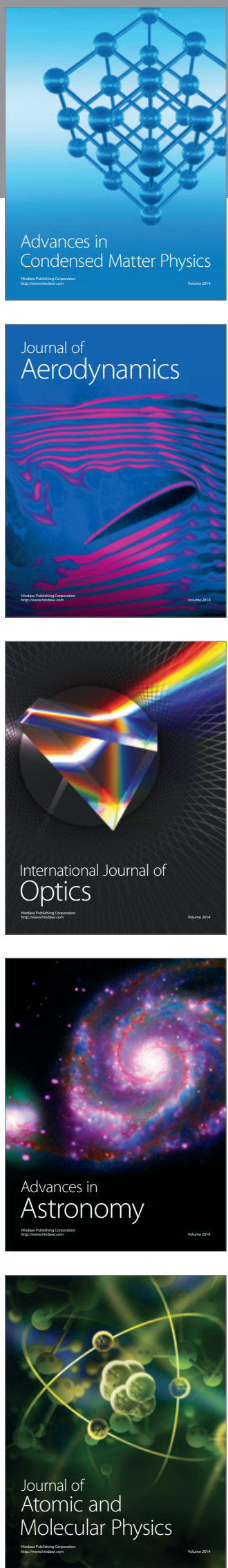